PROCEEDINGS OF THE AMERICAN MATHEMATICAL SOCIETY

Volume 124, Number 6, June 1996

\title{
ON THE DOUBLE COMMUTATION METHOD
}

\author{
F. GESZTESY AND G. TESCHL
}

(Communicated by Palle E. T. Jorgensen)

\begin{abstract}
We provide a complete spectral characterization of the double commutation method for general Sturm-Liouville operators which inserts any finite number of prescribed eigenvalues into spectral gaps of a given background operator. Moreover, we explicitly determine the transformation operator which links the background operator to its doubly commuted version (resulting in extensions and considerably simplified proofs of spectral results even for the special case of Schrödinger-type operators).
\end{abstract}

\section{INTRODUCTION}

Methods of inserting (and removing) eigenvalues in spectral gaps of a given onedimensional Schrödinger operator $H$ associated with differential expressions of the type $-\frac{d^{2}}{d x^{2}}+q$ on $(a, \infty), a \geq-\infty$, have recently attracted an enormous amount of attention. This is due to their prominent role in diverse fields such as the inverse scattering approach introduced by Deift and Trubowitz [7], supersymmetric quantum mechanics (see, e.g., [23] and the references therein), level comparison theorems (cf. [2] and the literature cited therein), as a tool to construct soliton solutions of the Korteweg-de Vries (KdV) hierarchy relative to (general) KdV background solutions (see, e.g., [3], [6], [7], [8], Ch. 4, [12], [15], [18], [23], [25]-[27], [30], Sect. 6.6, [33]-[36]), and in connection with Bäcklund transformations for the KdV hierarchy (cf., e.g., [10], [11], [13], [18], [20], [21], [23], [28], [29], [38]).

Historically, these methods of inserting eigenvalues go back to Jacobi [24] and Darboux [5] with decisive later contributions by Crum [4], Schmincke [34], and, especially, Deift [6]. Two particular methods, shortly to be discussed in an informal manner in (1.1)-(1.6) below, turned out to be of special importance: The single commutation method, also called the Crum-Darboux method [4], [5] (actually going back at least to Jacobi [24]), and the double commutation method, to be found, e.g., in the seminal work of Gel'fand and Levitan [16].

The single commutation method, although very simply implemented, has the distinct disadvantage of relying on positivity properties of certain solutions of $H \phi=$ $\lambda \phi$ which confines its applicability to the insertion of eigenvalues below the spectrum of $H$ (assuming $H$ to be bounded from below). A complete spectral characterization of this method has been provided by Deift [6] (see also [34]) on the basis of unitary

Received by the editors December 8, 1994.

1991 Mathematics Subject Classification. Primary 34B24, 34L05; Secondary 34B20, 47A10.

Key words and phrases. Commutation methods, Sturm-Liouville operators, eigenvalues.

(C)1996 American Mathematical Society 
equivalence of $\left.A^{*} A\right|_{\operatorname{Ker}(A)^{\perp}}$ and $\left.A A^{*}\right|_{\operatorname{Ker}\left(A^{*}\right)^{\perp}}$ for a densely defined closed linear operator $A$ in a (complex, separable) Hilbert space.

The double commutation method, on the other hand, allows one to insert eigenvalues into any spectral gap of $H$. Although relatively simply implemented also, a complete spectral characterization of the double commutation method for Schrödinger-type operators was only very recently achieved in [17] on the basis of WeylTitchmarsh $m$-function techniques. In this note we follow up on the double commutation method and provide, in particular,

- a complete spectral analysis of the double commutation method for general Sturm-Liouville operators on arbitrary intervals $(a, b),-\infty \leq a<b \leq \infty$.

- a functional analytic approach which not only avoids the Weyl-Titchmarsh theory employed in [17], but, in addition, considerably simplifies and streamlines the corresponding proofs in [17].

We emphasize that our formulation of the double commutation method for general Sturm-Liouville (SL) operators appears to be without precedent.

Before starting our analysis in Section 2, we informally describe the single and double commutation methods for general Sturm-Liouville differential expressions. Consider the differential expression $\left(p, p^{\prime}, k, k^{\prime} \in A C_{l o c}((a, b)), p, k>0\right.$, $\left.q \in L_{l o c}^{1}((a, b)),-\infty \leq a<b \leq \infty\right)$

$$
\tau=\frac{1}{k}\left(-\frac{d}{d x} p \frac{d}{d x}+q\right)
$$

and introduce

$$
a=\frac{1}{k}\left(\sqrt{k p} \frac{d}{d x}+\phi\right), \quad a^{*}=\frac{1}{k}\left(-\frac{d}{d x} \sqrt{k p}+\phi\right),
$$

where $\phi=(\sqrt{k p} \psi)^{\prime} / \psi$ and $\psi>0$ satisfies $\tau \psi=\lambda_{1} \psi$. A straightforward calculation reveals

$$
\tau=a a^{*}+\lambda_{1}, \quad \hat{\tau}=a^{*} a+\lambda_{1}=\frac{1}{k}\left(-\frac{d}{d x} p \frac{d}{d x}+\hat{q}\right)
$$

where

$$
\hat{q}=q-\frac{p^{\prime \prime}}{2}+\frac{\left(p^{\prime}\right)^{2}}{4 p}+\frac{3\left(k^{\prime}\right)^{2} p}{4 k^{2}}-\frac{k^{\prime \prime} p}{2 k}+\left(\frac{(k p)^{\prime}}{k}-2 \frac{d}{d x} p\right) \frac{d}{d x} \ln \psi
$$

Thus (taking proper domain considerations into account) we can define two operators $H, \hat{H}$ on $L^{2}((a, b) ; k d x)$ associated with $\tau, \hat{\tau}$ which are unitarily equivalent when restricted to the orthogonal complement of the eigenspaces corresponding to $\lambda_{1}$ [6] (cf. also [34]). Moreover, $H-\lambda_{1}, \hat{H}-\lambda_{1} \geq 0$, which is equivalent to the existence of the positive solution $\psi$ [22]. Formulas (1.1)-(1.4) constitute the single commutation method for general SL differential expressions as discussed, e.g., by Schmincke [34]. Next we assume that $\psi$ is square integrable near $a$ and consider two more expressions $a_{\gamma_{1}}, a_{\gamma_{1}}^{*}$ as above with $\psi_{\gamma_{1}}=\psi /\left(1+\gamma_{1} \int_{a}^{x} k(t) \psi(t)^{2} d t\right)$. This implies

$$
a^{*} a=a_{\gamma_{1}}^{*} a_{\gamma_{1}}, \quad \tau_{\gamma_{1}}=a_{\gamma_{1}} a_{\gamma_{1}}^{*}+\lambda_{1}=\frac{1}{k}\left(-\frac{d}{d x} p \frac{d}{d x}+q_{\gamma_{1}}\right),
$$

where

$$
q_{\gamma_{1}}=q+\left(\frac{(k p)^{\prime}}{k}-2 \frac{d}{d x} p\right) \frac{d}{d x} \ln \left(1+\gamma_{1} \int_{a}^{x} k(t) \psi(t)^{2} d t\right)
$$


Now observe that $q_{\gamma_{1}}$ is well defined even if $\psi$ has zeros, and hence we expect $H, H_{\gamma_{1}}$ (a SL operator associated with $\tau_{\gamma_{1}}$ ) to be closely related even in the case where all intermediate operators are ill-defined. This was first shown in [17] for the special case of Schrödinger-type operators where $k=p=1$. We shall prove this fact for general SL operators by explicitly computing a transformation operator for the pair $\tau, \tau_{\gamma_{1}}$ which, when restricted to the orthogonal complement of the eigenspace of $\lambda_{1}$, turns out to be unitary. Formulas (1.1)-(1.6) sum up the double commutation method for general SL differential expressions.

Our results are relevant in connection with inverse scattering theory in nonhomogeneous media (see, e.g., [1] and the references cited therein) and yield a direct construction of $N$-soliton solutions relative to arbitrary background solutions of the (generalized) KdV hierarchy along the methods of [18] (see also [20]).

\section{Construction of a transformation operator}

Let $k \in L_{\text {loc }}^{1}((a, b))$ with $k>0$. We pick $\mathfrak{H}=L^{2}((a, b) ; k d x)$ to be the underlying Hilbert space $(-\infty \leq a<b \leq \infty)$ and define for $c \in(a, b)$,

$$
L_{l o c}^{2}([a, b) ; k d x)=\left\{f \in L_{l o c}^{2}((a, b) ; k d x) \mid f \in L^{2}((a, c) ; k d x)\right\} .
$$

Next, choose a positive number $\gamma>0$, consider a fixed element $\psi \in L_{l o c}^{2}([a, b) ; k d x)$, and define the (linear) transformation

$$
\begin{array}{ll}
L_{l o c}^{2}([a, b) ; k d x) & \rightarrow L_{l o c}^{2}([a, b) ; k d x) \\
f(x) & \mapsto f_{\gamma}(x)=f(x)-\gamma \psi_{\gamma}(x) \int_{a}^{x} k(t) \overline{\psi(t)} f(t) d t,
\end{array}
$$

where $\psi_{\gamma}$ is defined by

$$
\psi_{\gamma}(x)=\frac{\psi(x)}{1+\gamma \int_{a}^{x} k(t)|\psi(t)|^{2} d t} .
$$

By inspection, the inverse transformation is given by

$$
\begin{aligned}
& U_{\gamma}^{-1}: L_{l o c}^{2}([a, b) ; k d x) \rightarrow L_{l o c}^{2}([a, b) ; k d x) \\
& g(x) \quad \mapsto g(x)+\gamma \psi(x) \int_{a}^{x} k(t) \overline{\psi_{\gamma}(t)} g(t) d t .
\end{aligned}
$$

The restriction of $U_{\gamma}$ to $\mathfrak{H}$ will be denoted by $U_{\gamma}$ as well. Note that we have

$$
1-\gamma \int_{a}^{x} k(t)\left|\psi_{\gamma}(t)\right|^{2} d t=\left(1+\gamma \int_{a}^{x} k(t)|\psi(t)|^{2} d t\right)^{-1}
$$

Lemma 2.1. The element $\psi_{\gamma}$ fulfills

$$
\psi_{\gamma} \in \mathfrak{H}, \quad\left\|\psi_{\gamma}\right\|^{2}=\frac{1}{\gamma}\left(1-\lim _{x \rightarrow b}\left(1+\gamma \int_{a}^{x} k(t)|\psi(t)|^{2} d t\right)^{-1}\right) .
$$

Denote by $P, P_{\gamma}$ the orthogonal projections onto the one-dimensional subspaces of $\mathfrak{H}$ spanned by $\psi, \psi_{\gamma}($ set $P=0$ if $\psi \notin \mathfrak{H})$; then the operator $U_{\gamma}$ is unitary from $(1-P) \mathfrak{H}$ onto $\left(1-P_{\gamma}\right) \mathfrak{H}$.

Proof. The claims concerning $\psi_{\gamma}$ are straightforward. Next we note that for $x \in$ $(a, b)$ and $f, f_{\gamma}$ as in $(2.2)$,

$$
\int_{a}^{x} k(t) \overline{\psi_{\gamma}(t)} f_{\gamma}(t) d t=\frac{\int_{a}^{x} k(t) \overline{\psi(t)} f(t) d t}{1+\gamma \int_{a}^{x} k(t)|\psi(t)|^{2} d t} .
$$


A direct calculation then shows

$$
\int_{a}^{x} k(t)\left|f_{\gamma}(t)\right|^{2} d t=\int_{a}^{x} k(t)|f(t)|^{2} d t-\gamma \frac{\left|\int_{a}^{x} k(t) \overline{\psi(t)} f(t) d t\right|^{2}}{1+\gamma \int_{a}^{x} k(t)|\psi(t)|^{2} d t}
$$

which proves the lemma if $\psi \in \mathfrak{H}$. Otherwise, consider $U_{\gamma}, U_{\gamma}^{-1}$ on the dense subspace of square integrable elements with compact support in $(a, b)$ and take closures (cf., e.g., [39], Theorem 6.13).

Using, e.g., the polarization identity, we obtain in addition

$$
\begin{aligned}
\int_{a}^{x} k(t) \overline{g_{\gamma}(t)} f_{\gamma}(t) d t= & \int_{a}^{x} k(t) \overline{g(t)} f(t) d t \\
& -\gamma \frac{\int_{a}^{x} k(t) \overline{\psi(t)} f(t) d t \int_{a}^{x} k(t) \psi(t) \overline{g(t)} d t}{1+\gamma \int_{a}^{x} k(t)|\psi(t)|^{2} d t} .
\end{aligned}
$$

\section{InSERTING A SINGLE EIGENVALUE}

Now we turn to the SL differential expression

$$
\tau=\frac{1}{k}\left(-\frac{d}{d x} p \frac{d}{d x}+q\right)
$$

where the coefficients $p, q, k$ are real-valued satisfying

$$
p^{-1}, q, k \in L_{l o c}^{1}((a, b)), \quad k>0, \quad k p \in A C_{l o c}((a, b)) .
$$

We are interested in self-adjoint operators $H$ associated with $\tau$ and separated boundary conditions.

Hypothesis (H.3.1). Let $\lambda \in \mathbb{R}$ and suppose $\psi$ satisfies the following conditions.

(i) $\psi, p \psi^{\prime} \in A C_{l o c}((a, b))$ and $\psi$ is a real-valued solution of $\tau \phi=\lambda \phi$.

(ii) $\psi$ is square integrable near $a$ and fulfills the boundary condition (of $H$ ) at $a$ and $b$ if any (i.e., if $\tau$ is limit circle (l.c.) at $a$ respectively $b$ ).

Sufficient conditions for the existence of a function $\psi$ satisfying (H.3.1) are

(a) $\lambda_{1} \in \sigma_{p}(H)\left(\sigma_{p}(\right.$.$) the point spectrum, i.e., the set of eigenvalues), or$

(b) $\tau$ is l.c. at $a$ but not at $b$, or

(c) $\sigma(H) \neq \mathbb{R}$ (and $\lambda \in \mathbb{R} \backslash \sigma(H)$ ), or

(d) $\sigma\left(H_{-}^{c}\right) \neq \mathbb{R}$ (and $\lambda \in \mathbb{R} \backslash \sigma\left(H_{-}^{c}\right)$ ) where $H_{-}^{c}$ is a restriction of $H$ to $L^{2}((a, c)$ ) with $c \in(a, b)$ (finite) and, e.g., a Dirichlet boundary condition at $c$.

If $\lambda_{1} \in \mathbb{R}$ and $\psi\left(\lambda_{1},.\right)$ obeys (H.3.1), it follows that $H$ is explicitly given by

$$
\begin{aligned}
H f=\tau f, \quad \mathfrak{D}(H)= & \left\{f \in \mathfrak{H} \mid f, p f^{\prime} \in A C_{\text {loc }}((a, b)) ; \tau f \in \mathfrak{H} ;\right. \\
& W_{a}\left(\psi\left(\lambda_{1}\right), f\right)=0 \text { if } \tau \text { is l.c. at } a \\
& \left.W_{b}\left(\psi\left(\lambda_{1}\right), f\right)=0 \text { if } \tau \text { is l.c. at } b\right\}
\end{aligned}
$$

with $W_{x}(u, v)=p(x)\left(u(x) v(x)^{\prime}-u(x)^{\prime} v(x)\right)$ the (modified) Wronskian of $u, v \in$ $A C_{l o c}((a, b))$.

We now use Lemma 2.1 with $\psi=\psi\left(\lambda_{1}\right), \gamma=\gamma_{1}$ to prove

Theorem 3.2. Let $\lambda_{1} \in \mathbb{R}$ and $\psi\left(\lambda_{1},.\right)$ be a solution satisfying (H.3.1). Define $U_{\gamma_{1}}, P\left(\lambda_{1}\right), P_{\gamma_{1}}\left(\lambda_{1}\right)$ as in (2.2) and Lemma 2.1 in terms of $\psi\left(\lambda_{1},.\right)$ and set $\psi_{\gamma_{1}}\left(\lambda_{1},.\right)=U_{\gamma_{1}} \psi\left(\lambda_{1},.\right)$. Then the operator $H_{\gamma_{1}}$ defined by

$$
\begin{aligned}
H_{\gamma_{1}} f=\tau_{\gamma_{1}} f, \quad \mathfrak{D}\left(H_{\gamma_{1}}\right)= & \left\{f \in \mathfrak{H} \mid f, p f^{\prime} \in A C_{l o c}((a, b)) ; \tau_{\gamma_{1}} f \in \mathfrak{H} ;\right. \\
& \left.W_{a}\left(\psi_{\gamma_{1}}\left(\lambda_{1}\right), f\right)=W_{b}\left(\psi_{\gamma_{1}}\left(\lambda_{1}\right), f\right)=0\right\},
\end{aligned}
$$


with

$$
q_{\gamma_{1}}=q+\left(\frac{(k p)^{\prime}}{k}-2 \frac{d}{d x} p\right) \frac{d}{d x} \ln \left(1+\gamma_{1} \int_{a}^{x} k(t) \psi\left(\lambda_{1}, t\right)^{2} d t\right)
$$

is self-adjoint. Moreover, $H_{\gamma_{1}}$ has the eigenfunction $\psi_{\gamma_{1}}\left(\lambda_{1}\right)=U_{\gamma_{1}} \psi\left(\lambda_{1}\right)$ associated with the eigenvalue $\lambda_{1}$. If $\psi\left(\lambda_{1}\right) \notin \mathfrak{H}$ (and hence $\tau$ is limit point (l.p.) at b) we have

$$
H_{\gamma_{1}}\left(1-P_{\gamma_{1}}\left(\lambda_{1}\right)\right)=U_{\gamma_{1}} H U_{\gamma_{1}}^{-1}\left(1-P_{\gamma_{1}}\left(\lambda_{1}\right)\right)
$$

and thus

$$
\begin{aligned}
& \sigma\left(H_{\gamma_{1}}\right)=\sigma(H) \cup\left\{\lambda_{1}\right\}, \quad \sigma_{a c}\left(H_{\gamma_{1}}\right)=\sigma_{a c}(H), \\
& \sigma_{p}\left(H_{\gamma_{1}}\right)=\sigma_{p}(H) \cup\left\{\lambda_{1}\right\}, \quad \sigma_{s c}\left(H_{\gamma_{1}}\right)=\sigma_{s c}(H) .
\end{aligned}
$$

(Here $\sigma_{a c}(),. \sigma_{s c}($.$) denotes the absolutely and singularly continuous spectrum, re-$ spectively.) If $\psi\left(\lambda_{1}\right) \in \mathfrak{H}$ there is a unitary operator $\tilde{U}_{\gamma_{1}}=U_{\gamma_{1}} \oplus \sqrt{1+\gamma_{1}\left\|\psi\left(\lambda_{1}\right)\right\|^{2}} \mathbf{1}$ on $\left(1-P\left(\lambda_{1}\right)\right) \mathfrak{H} \oplus P\left(\lambda_{1}\right) \mathfrak{H}$ such that

$$
H_{\gamma_{1}}=\tilde{U}_{\gamma_{1}} H \tilde{U}_{\gamma_{1}}^{-1} \text {. }
$$

Proof. It suffices to prove $H_{\gamma_{1}}\left(1-P_{\gamma_{1}}\left(\lambda_{1}\right)\right)=U_{\gamma_{1}} H U_{\gamma_{1}}^{-1}\left(1-P_{\gamma_{1}}\left(\lambda_{1}\right)\right)$. Let $f$ be square integrable near $a$ (with $f, p f^{\prime} \in A C_{l o c}((a, b))$ ) such that $\tau f$ is also square integrable near $a$ and $f$ fulfills the boundary condition at $a$ (if any). Then a straightforward calculation shows

$$
\tau_{\gamma_{1}}\left(U_{\gamma_{1}} f\right)=U_{\gamma_{1}}(\tau f)
$$

and we only have to check the boundary conditions at $a$ and $b$. Equation (2.8) shows that $\tau_{\gamma_{1}}$ is l.c. at $a$, respectively $b$, if $\tau$ is. The formula

$$
W_{x}\left(\psi_{\gamma_{1}}\left(\lambda_{1}\right), U_{\gamma_{1}} f\right)=\frac{W_{x}\left(\psi\left(\lambda_{1}\right), f\right)}{1+\gamma_{1} \int_{a}^{x} k(t) \psi\left(\lambda_{1}, t\right)^{2} d t}
$$

reveals $W_{a}\left(\psi_{\gamma_{1}}\left(\lambda_{1}\right), U_{\gamma_{1}} f\right)=0$ for $f \in \mathfrak{D}(H)$. Furthermore, we claim that

$$
W_{b}\left(\psi_{\gamma_{1}}\left(\lambda_{1}\right), U_{\gamma_{1}} f\right)=0, \quad f \in \mathfrak{D}(H) .
$$

This is clear if $\psi\left(\lambda_{1}\right) \in \mathfrak{H}$, otherwise, i.e., if $\psi\left(\lambda_{1}\right) \notin \mathfrak{H}$ we use

$$
\left|W_{x}\left(\psi_{\gamma_{1}}\left(\lambda_{1}\right), U_{\gamma_{1}} f\right)\right|^{2}=\frac{\left|\int_{a}^{x} k(t) \psi\left(\lambda_{1}, t\right)\left(\lambda_{1}-\tau\right) f(t) d t\right|^{2}}{\left(1+\gamma_{1} \int_{a}^{x} k(t) \psi\left(\lambda_{1}, t\right)^{2} d t\right)^{2}} .
$$

The right-hand side of (3.12) tends to zero for $f \in \mathfrak{D}(H)$ as can be seen from (2.8) and the fact that $U_{\gamma_{1}}$ is unitary. Combining the last results yields

$$
\left(1-P_{\gamma_{1}}\left(\lambda_{1}\right)\right) U_{\gamma_{1}} \mathfrak{D}(H) \subseteq\left(1-P_{\gamma_{1}}\left(\lambda_{1}\right)\right) \mathfrak{D}\left(H_{\gamma_{1}}\right) .
$$

But $\left(1-P_{\gamma_{1}}\left(\lambda_{1}\right)\right) U_{\gamma_{1}} \mathfrak{D}(H)$ cannot be properly contained in $\left(1-P_{\gamma_{1}}\left(\lambda_{1}\right)\right) \mathfrak{D}\left(H_{\gamma_{1}}\right)$ by the property of self-adjoint operators being maximal.

Remark 3.3. (i) If $H$ has an eigenfunction $\psi\left(\lambda_{1}\right)$ we can remove this eigenfunction from the spectrum upon choosing $\gamma_{1}=-\left\|\psi\left(\lambda_{1}\right)\right\|^{-2}$. Then the corresponding element $\psi_{\gamma_{1}}\left(\lambda_{1}\right)$ is not in $\mathfrak{H}$, implying that $\tau_{\gamma_{1}}$ is l.p. at $b$.

(ii) The double commutation method has the pleasant feature of leaving $p$ invariant. This is in sharp contrast to the double commutation method for Jacobi operators [19] (the finite difference analog for (3.1)). let

(iii) Let $u$ (with $u, p u^{\prime} \in A C_{l o c}((a, b))$ ) fulfill $\tau u=z u$ (with $z \in \mathbb{C} \backslash\left\{\lambda_{1}\right\}$ ), and

$$
v(z, x)=u(z, x)+\frac{\gamma_{1}}{z-\lambda_{1}} \psi_{\gamma_{1}}\left(\lambda_{1}, x\right) W_{x}\left(\psi\left(\lambda_{1}\right), u(z)\right) .
$$


Then $v, p v^{\prime} \in A C_{l o c}((a, b))$ and $v$ fulfills $\tau_{\gamma_{1}} v=z v$. If $u$ is square integrable near $a$ and fulfills the boundary condition at $a$ (if any) we have $v=U_{\gamma_{1}} u$. We also note

$$
|v(z, x)|^{2}=|u(z, x)|^{2}-\frac{\gamma_{1} k(x)^{-1}}{\left|z-\lambda_{1}\right|^{2}} \frac{d}{d x}\left(\frac{\left|W_{x}\left(\psi\left(\lambda_{1}\right), u(z)\right)\right|^{2}}{1+\gamma_{1} \int_{a}^{x} k(t) \psi\left(\lambda_{1}, t\right)^{2} d t}\right),
$$

and if $\hat{u}, \hat{v}$ are constructed analogously, then

$$
\begin{aligned}
W_{x}(v(z), \hat{v}(\hat{z}))= & W_{x}(u(z), \hat{u}(\hat{z}))-\frac{\gamma_{1}}{1+\gamma_{1} \int_{a}^{x} k(t) \psi\left(\lambda_{1}, t\right)^{2} d t} \times \\
& \frac{z-\hat{z}}{\left(z-\lambda_{1}\right)\left(\hat{z}-\lambda_{1}\right)} W_{x}\left(\psi\left(\lambda_{1}\right), u(z)\right) W_{x}\left(\psi\left(\lambda_{1}\right), \hat{u}(\hat{z})\right) .
\end{aligned}
$$

(iv) Writing $U_{\gamma_{1}} f$ as

$$
\left(U_{\gamma_{1}} f\right)(x)=f(x)-\int_{a}^{x} \frac{\gamma_{1} k(t) \psi\left(\lambda_{1}, x\right) \psi\left(\lambda_{1}, t\right)}{1+\gamma_{1} \int_{a}^{x} k(s) \psi\left(\lambda_{1}, s\right)^{2} d s} f(t) d t,
$$

we see that $U_{\gamma_{1}}$ is the transformation operator for $H, H_{\gamma_{1}}$ in the terminology of [30] and [31].

(v) The limiting case $\gamma=\infty$ can be handled analogously, producing a unitarily equivalent operator.

Finally we discuss conditions for $\tau_{\gamma_{1}}$ to be $l . p$. at $a, b$. Let $c \in(a, b)$ and let $H_{+}^{c}$ denote a self-adjoint operator associated with $\tau$ on $(c, b)$ and the boundary condition induced by $\psi\left(\lambda_{1}\right)$ at $c$ (i.e., $\left.W_{c}\left(f, \psi\left(\lambda_{1}\right)\right)=0, f \in \mathfrak{D}\left(H_{+}^{c}\right)\right)$.

Hypothesis (H.3.4). Suppose one of the following spectral conditions (i)-(iii) holds.

(i) $\sigma_{\text {ess }}\left(H_{+}^{c}\right) \neq \emptyset$.

(ii) $\sigma\left(H_{+}^{c}\right)=\sigma_{d}\left(H_{+}^{c}\right)=\left\{\lambda_{n}\right\}_{n \in \mathbb{Z}}$ with $\sum_{n \in \mathbb{Z}}\left(1+\lambda_{n}^{2}\right)^{-1}=\infty$.

(iii) $H_{+}^{c}$ is bounded from below and $\int^{b}|k(x) / p(x)|^{1 / 2} d x=\infty$.

All conditions (i)-(iii) imply that $\tau$ is l.p. at $b$. This is clear for (i), (ii) since if $H_{+}^{c}$ were $l . c$. at $b$ its resolvent would be a Hilbert-Schmidt operator contradicting (i), (ii). For (iii) this follows from [17], Lemma C.1.

Theorem 3.5. (i) $\tau_{\gamma_{1}}$ is l.p. at a if and only if $\tau$ is and l.c. at $b$ if $\tau$ is.

(ii) Assume (H.3.4), Then $\tau_{\gamma_{1}}$ is l.p. at b.

Proof. (i) follows from (3.15). For (ii) consider the doubly commuted operator $H_{+, \gamma_{1}^{c}}^{c}$ of $H_{+}^{c}$, where $\gamma_{1}^{c}=\gamma_{1} /\left(1+\gamma_{1} \int_{a}^{c} \psi\left(\lambda_{1}, t\right)^{2} d t\right)$. Then $\left.\tau_{\gamma_{1}}\right|_{(c, b)}=\tau_{\gamma_{1}^{c}}^{c}$ and $H_{+, \gamma_{1}^{c}}^{c}$ also satisfies (H.3.4). Hence $\tau_{\gamma_{1}}$ is l.p. at $b$ as claimed.

Remark 3.6. (i) Removing an eigenvalue (cf. Remark 3.3 (i)) from an operator which is l.c. at $b$ yields an operator which is $l . p$. Thus $\tau_{\gamma_{1}}$ is not necessarily l.p. if $\tau$ is. Moreover, this shows that one cannot insert additional eigenvalues into an operator which is l.c. at $b$ (remove this eigenvalue again to obtain a contradiction).

(ii) Clearly we can interchange the role of $a$ and $b$. One only has to substitute $a \rightarrow b$ in the text and $\int_{a} \rightarrow \int^{b}$ in the formulas.

(iii) As long as $\tau$ is l.c. at $a$ our method can be used to insert additional eigenvalues into the spectrum of $H$. In the special case of Schrödinger operators where $k=p=1$ this was first observed by Gel'fand and Levitan [16] in connection with 
Wigner-von Neumann examples. More recent studies can be found in [9], [32], [37] (see also [8], Section 4.4).

(iv) Theorems 3.2 and 3.5 were first derived in [17] for the special case $p=k=1$ and under somewhat more restrictive spectral conditions (such as $\lambda_{1} \in \mathbb{R} \backslash \sigma(H)$ ).

\section{INSERTING FINITELY MANY EIGENVALUES}

Finally we demonstrate how to iterate this method. We choose a given background operator $H$ (with coefficients $k, p, q$ ) and pick $\gamma_{1}>0, \lambda_{1} \in \mathbb{R}$. Now choose $\psi\left(\lambda_{1}\right)$, as in Section 3 to define the transformation $U_{\gamma_{1}}$ and the operator $H_{\gamma_{1}}$. Next we choose $\gamma_{2}>0, \lambda_{2} \in \mathbb{R}$ and another function $\psi\left(\lambda_{2}\right)$ to define $\psi_{\gamma_{1}}\left(\lambda_{2}\right)=U_{\gamma_{1}} \psi\left(\lambda_{2}\right)$ and corresponding operators $U_{\gamma_{1}, \gamma_{2}}$ and $H_{\gamma_{1}, \gamma_{2}}$. Applying this procedure $N$ times leads to

Theorem 4.1. Let $H$ be the background operator (3.3), and let $\gamma_{j}>0, \lambda_{j} \in \mathbb{R}$, $1 \leq j \leq N$, be such that there exist corresponding solutions $\psi\left(\lambda_{j}, x\right)$ of $\tau \phi=$ $\lambda_{j} \phi$ satisfying (H.3.1). We set $\psi_{\gamma_{1}, \ldots, \gamma_{\ell}}\left(\lambda_{j}\right)=U_{\gamma_{1}, \ldots, \gamma_{\ell}} \cdots U_{\gamma_{1}} \psi\left(\lambda_{j}\right)$ and define the following matrices $(1 \leq \ell \leq N)$

$$
\begin{gathered}
C_{\ell}(x)=\left\{\delta_{r, s}+\sqrt{\gamma_{r} \gamma_{s}} \int_{a}^{x} k(t) \psi\left(\lambda_{r}, t\right) \psi\left(\lambda_{s}, t\right) d t\right\}_{1 \leq r, s \leq \ell}, \\
C_{\ell ; i, j}(x)=\left\{\begin{array}{cc}
C_{\ell-1}(x)_{r, s}, & r, s \leq \ell-1 \\
\sqrt{\gamma_{s}} \int_{a}^{x} k(t) \psi\left(\lambda_{i}, t\right) \psi\left(\lambda_{s}, t\right) d t, & s \leq \ell-1, r=\ell \\
\sqrt{\gamma_{r}} \int_{a}^{x} k(t) \psi\left(\lambda_{r}, t\right) \psi\left(\lambda_{j}, t\right) d t, & r \leq \ell-1, s=\ell \\
\int_{a}^{x} k(t) \psi\left(\lambda_{i}, t\right) \psi\left(\lambda_{j}, t\right) d t, & r=s=\ell
\end{array}\right]_{1 \leq r, s \leq \ell}, \\
\Psi_{\ell}\left(\lambda_{j}, x\right)=\left\{\begin{array}{cc}
C_{\ell}(x)_{r, s}, & r, s \leq \ell \\
\sqrt{\gamma_{s}} \int_{a}^{x} k(t) \psi\left(\lambda_{j}, t\right) \psi\left(\lambda_{s}, t\right) d t, & s \leq \ell, r=\ell+1 \\
\sqrt{\gamma_{r}} \psi\left(\lambda_{r}, x\right), & r \leq \ell, s=\ell+1 \\
\psi\left(\lambda_{j}, x\right), & r=s=\ell+1
\end{array}\right.
\end{gathered} .
$$

Then we have $\left(\operatorname{set} C_{0}(x)=1\right)$

$$
1+\gamma_{\ell} \int_{a}^{x} k(t) \psi_{\gamma_{1}, \ldots, \gamma_{\ell}}\left(\lambda_{\ell}, t\right)^{2} d t=\frac{\operatorname{det} C_{\ell}(x)}{\operatorname{det} C_{\ell-1}(x)}
$$

and

$$
q_{\gamma_{1}, \ldots, \gamma_{N}}=q+\left(\frac{(k p)^{\prime}}{k}-2 \frac{d}{d x} p\right) \frac{d}{d x} \ln \operatorname{det} C_{N} .
$$

Furthermore, we obtain

$$
\int_{a}^{x} k(t) \psi_{\gamma_{1}, \ldots, \gamma_{\ell}}\left(\lambda_{i}, t\right) \psi_{\gamma_{1}, \ldots, \gamma_{\ell}}\left(\lambda_{j}, t\right) d t=\frac{\operatorname{det} C_{\ell ; i, j}(x)}{\operatorname{det} C_{\ell-1}(x)}
$$

and

$$
\psi_{\gamma_{1}, \ldots, \gamma_{\ell}}\left(\lambda_{j}, x\right)=\frac{\operatorname{det} \Psi_{\ell}\left(\lambda_{j}, x\right)}{\operatorname{det} C_{\ell}(x)}
$$


The spectrum of $H_{\gamma_{1}, \ldots, \gamma_{N}}$ is given by

$$
\begin{array}{rlll}
\sigma\left(H_{\gamma_{1}, \ldots, \gamma_{N}}\right) & =\sigma(H) \cup\left\{\lambda_{j}\right\}_{j=1}^{N}, & \sigma_{a c}\left(H_{\gamma_{1}, \ldots, \gamma_{N}}\right) & =\sigma_{a c}(H), \\
\sigma_{p}\left(H_{\gamma_{1}, \ldots, \gamma_{N}}\right) & =\sigma_{p}(H) \cup\left\{\lambda_{j}\right\}_{j=1}^{N}, & \sigma_{s c}\left(H_{\gamma_{1}, \ldots, \gamma_{N}}\right) & =\sigma_{s c}(H) .
\end{array}
$$

Moreover,

$$
\begin{aligned}
& H_{\gamma_{1}, \ldots, \gamma_{N}}\left(1-\sum_{j=1}^{N} P_{\gamma_{1}, \ldots, \gamma_{N}}\left(\lambda_{j}\right)\right) \\
& \quad=\left(U_{\gamma_{1}, \ldots, \gamma_{N}} \cdots U_{\gamma_{1}}\right) H\left(U_{\gamma_{1}}^{-1} \cdots U_{\gamma_{1}, \ldots, \gamma_{N}}^{-1}\right)\left(1-\sum_{j=1}^{N} P_{\gamma_{1}, \ldots, \gamma_{N}}\left(\lambda_{j}\right)\right),
\end{aligned}
$$

where $P_{\gamma_{1}, \ldots, \gamma_{N}}\left(\lambda_{j}\right)$ denotes the projection onto the one-dimensional subspace spanned by $\psi_{\gamma_{1}, \ldots, \gamma_{N}}\left(\lambda_{j}\right)$.

Proof. It suffices to prove (4.6), (4.7) which requires a straightforward induction argument using Sylvester's determinant identity ([14], Sect. II.3). The resulting identity

$$
\begin{aligned}
\operatorname{det} C_{\ell}(x) \operatorname{det} C_{\ell ; i, j}(x) & -\gamma_{\ell} \operatorname{det} C_{\ell ; \ell, j}(x) \operatorname{det} C_{\ell ; i, \ell}(x) \\
& =\operatorname{det} C_{\ell-1}(x) \operatorname{det} C_{\ell+1 ; i, j}(x),
\end{aligned}
$$

together with (2.9) then proves (4.6). Similarly,

$$
\begin{array}{r}
\operatorname{det} C_{\ell}(x) \operatorname{det} \Psi_{\ell-1}\left(\lambda_{j}, x\right)-\gamma_{\ell} \operatorname{det} \Psi_{\ell-1}\left(\lambda_{\ell}, x\right) \operatorname{det} C_{\ell ; \ell, j}(x) \\
=\operatorname{det} C_{\ell-1}(x) \operatorname{det} \Psi_{\ell}\left(\lambda_{j}, x\right),
\end{array}
$$

and (2.2) prove (4.7). The rest then follows from these two equations and Theorem 3.2 .

Remark 4.2. (i) For any element $f$ which is square integrable near $-\infty, f_{\gamma_{1}, \ldots, \gamma_{\ell}}=$ $U_{\gamma_{1}, \ldots, \gamma_{\ell}} \cdots U_{\gamma_{1}} f$ is given by substituting $\psi\left(\lambda_{j}\right) \rightarrow f$ in (4.7). Likewise we get the scalar product of $f_{\gamma_{1}, \ldots, \gamma_{\ell}}$ and $g_{\gamma_{1}, \ldots, \gamma_{\ell}}$ from (4.6) by substituting $\psi\left(\lambda_{i}\right) \rightarrow f$ and $\psi\left(\lambda_{j}\right) \rightarrow g$ in $(4.2)$.

(ii) Equation (4.7) can be rephrased as

$$
\begin{aligned}
& \left(\sqrt{\gamma_{1}} \psi_{\gamma_{1}, \ldots, \gamma_{N}}\left(\lambda_{1}, x\right), \ldots, \sqrt{\gamma_{N}} \psi_{\gamma_{1}, \ldots, \gamma_{N}}\left(\lambda_{N}, x\right)\right) \\
& \quad=\left(C_{N}(x)\right)^{-1}\left(\sqrt{\gamma_{1}} \psi\left(\lambda_{1}, x\right), \ldots, \sqrt{\gamma_{N}} \psi\left(\lambda_{N}, x\right)\right),
\end{aligned}
$$

where $\left(C_{N}(x)\right)^{-1}$ denotes the inverse of $C_{N}(x)$.

Clearly Theorem 3.5 extends (by induction) to this more general situation.

Theorem 4.3. (i) $\tau_{\gamma_{1}, \ldots, \gamma_{N}}$ is l.p. at $a$ if and only if $\tau$ is and l.c. at $b$ if $\tau$ is.

(ii) Assume (H.3.4). Then $\tau_{\gamma_{1}, \ldots, \gamma_{N}}$ is l.p. at b.

\section{REFERENCES}

[1] T. Aktosun, M. Klaus, and C. van der Mee, Scattering and inverse scattering in onedimensional nonhomogeneous media, J. Math. Phys. 32, 1717-1744 (1992). MR 93g:81200

[2] B. Baumgartner, Level comparison theorems, Ann. Phys. (N.Y.) 168, 484-526 (1986). MR

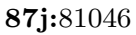

[3] R. F. Bikbaev and R. A. Sharipov, Asymptotics at $t \rightarrow \infty$ of the solution to the Cauchy problem for the Korteweg-de Vries equation in the class of potentials with finite-gap behavior as $x \rightarrow \pm \infty$, Theoret. Math. Phys. 78, 244-252 (1989). MR 90i:35227

[4] M. M. Crum, Associated Sturm-Liouville systems, Quart. J. Math. Oxford (2) 6, 121-127 (1955). MR 17:266g 
[5] G. Darboux, Sur une proposition relative aux équations linéaires, C. R. Acad. Sci. (Paris) 94, 1456-1459 (1882).

[6] P. A. Deift, Applications of a commutation formula, Duke Math. J. 45, 267-310 (1978). MR 81g:47001

[7] P. Deift and E. Trubowitz, Inverse scattering on the line, Commun. Pure Appl. Math. 32, 121-251 (1979). MR 80e:34011

[8] M. S. P. Eastham and H. Kalf, Schrödinger-Type Operators with Continuous Spectra, Pitman, Boston, 1982. MR 84i:35107

[9] M. S. P. Eastham and J. B. McLeod, The existence of eigenvalues embedded in the continuous spectrum of ordinary differential operators, Proc. Roy. Soc. Edinburgh 79A, 25-34 (1977). MR 57:16791

[10] F. Ehlers and H. Knörrer, An algebro-geometric interpretation of the Bäcklund transformation of the Korteweg-de Vries equation, Comment. Math. Helv. 57, 1-10 (1982). MR 84b:14022

[11] N. M. Ercolani and H. Flaschka, The geometry of the Hill equation and of the Neumann system, Phil. Trans. Roy. Soc. London A315, 405-422 (1985). MR 87f:58064

[12] N. E. Firsova, On solution of the Cauchy problem for the Korteweg-de Vries equation with initial data the sum of a periodic and a rapidly decreasing function, Math. USSR Sbornik 63, 257-265 (1989). MR 90a:35197

[13] H. Flaschka and D. W. McLaughlin, Some comments on Bäcklund transformations, canonical transformations, and the inverse scattering method, in "Bäcklund Transformations, the Inverse Scattering Method, Solitons, and their Applications", R. M. Miura (ed.), Lecture Notes in Math. 515, Springer, Berlin, 1976, p. 252-295. MR 58:29425

[14] F. R. Gantmacher, The Theory of Matrices, Vol. 1, Chelsa, New York, 1990.

[15] C. S. Gardner, J. M. Greene, M. D. Kruskal, and R. M. Miura, Korteweg-de Vries equation and generalizations. VI. Methods for exact solution, Commun. Pure Appl. Math. 27, 97-133 (1974). MR 49:898

[16] I. M. Gel'fand and B. M. Levitan, On the determination of a differential equation from its spectral function, Amer. Math. Soc. Transl. Ser 2, 1, 253-304 (1955). MR 17:489c

[17] F. Gesztesy, A complete spectral characterization of the double commutation method, J. Funct. Anal. 117, 401-446 (1993). MR 94m:47093

[18] F. Gesztesy and R. Svirsky, ( $m$ )KdV-Solitons on the background of quasi-periodic finite-gap solutions, Memoirs Amer. Math. Soc. 118, No. 563 (1995). CMP 95:04

[19] F. Gesztesy and G. Teschl, Commutation methods for Jacobi operators, J. Differential Equations (to appear).

[20] F. Gesztesy and K. Unterkofler, Isospectral deformations for Sturm-Liouville and Dirac-type operators and associated nonlinear evolution equations, Rep. Math. Phys. 31, 113-137 (1992). MR 94f: 35124

[21] F. Gesztesy and R. Weikard, Spectral deformations and soliton equations, in "Differential Equations with Applications in Mathematical Physics", W. F. Ames, E. M. Harrell, J. V. Herod (eds.), Academic Press, Boston, 1993, p. 101-139. MR 93m:34138

[22] F. Gesztesy and Z. Zhao, On critical and subcritical Sturm-Liouville operators, J. Funct. Anal. 98, 311-345 (1991). MR 93f:34146

[23] F. Gesztesy, W. Schweiger, and B. Simon, Commutation methods applied to the mKdVequation, Trans. Amer. Math. Soc. 324, 465-525 (1991). MR 92b:35132

[24] C. G. J. Jacobi, Zur Theorie der Variationsrechnung und der Differentialgleichungen, J. Reine Angew. Math. 17, 68-82 (1837).

[25] I. Kay and H. E. Moses, Reflectionless transmission through dielectrics and scattering potentials, J. Appl. Phys. 27, 1503-1508 (1956). MR 19:805e

[26] I. M. Krichever, Potentials with zero coefficient of reflection on a background of finite-zone potentials, Funct. Anal. Appl. 9, 161-163 (1975).

[27] E. A. Kuznetsov and A. V. Mikhailov, Stability of solitary waves in nonlinear weakly dispersive media, Sov. Phys. JETP 40, 855-859 (1975). MR 52:8685

[28] H. P. McKean, Geometry of KdV (1): Addition and the unimodular spectral classes, Rev. Mat. Iberoamericana 2, 235-261 (1986). MR 89b:58096

[29] H. P. McKean, Geometry of KdV (2): Three examples, J. Stat. Phys. 46, 1115-1143 (1987). MR 89b:58097 
[30] B. M. Levitan, Inverse Sturm-Liouville Problems, VNU Science Press, Utrecht, 1987. MR 89b:34001

[31] V. A. Marchenko, Sturm-Liouville Operators and Applications, Birkhäuser, Basel, 1986.

[32] R. R. del Rio Castillo, Embedded eigenvalues of Sturm Liouville operators, Commun. Math. Phys. 142, 421-431 (1991). MR 92h:47067

[33] A. V. Rybin and M. A. Sall', Solitons of the Korteweg-de Vries equation on the background of a known solution, Theoret. Math. Phys. 63, 545-550 (1985). MR 86k:35145

[34] U. -W. Schmincke, On Schrödinger's factorization method for Sturm-Liouville operators, Proc. Roy. Soc. Edinburgh 80A, 67-84 (1978). MR 80f:34025

[35] R. A. Sharipov, Finite-zone analogues of $N$-multiplet solutions of the Korteweg-de Vries equation, Russ. Math. Surv. 41:5, 165-166 (1986). MR 88e:35177

[36] R. A. Sharipov, Soliton multiplets of the Korteweg-de Vries equation, Sov. Phys. Dokl. 32, 121-123 (1987).

[37] C. Thurlow, The point-continuous spectrum of second order, ordinary differential operators, Proc. Roy. Soc. Edinburgh 84A, 197-211 (1979). MR 81c:34018

[38] H. D. Wahlquist, Bäcklund transformations of potentials of the Korteweg-de Vries equation and the interaction of solitons with cnoidal waves, in "Bäcklund Transformations, the Inverse Scattering Method, Solitons, and their Applications", R. M. Miura (ed.), Lecture Notes in Math. 515, Springer, Berlin, 1976, p. 162-183. MR 58:29470

[39] J. Weidmann, Linear Operators in Hilbert Spaces, Springer, New York, 1980. MR 81e:47001

(F. Gesztesy and G. Teschl) Department of Mathematics, University of Missouri, ColumBIA, Missouri 65211

E-mail address: mathfg@mizzou1.missouri.edu

E-mail address: mathgr42@mizzou1.missouri.edu

(G. Teschl) Department of Theoretical Physics, Technical University of Graz, Graz, 8010, Austria

Current address, G. Teschl: Institut für Reine und Angewandte Mathematik, RheinischWestfälische Technische Hochschule Aachen, D-52056 Aachen, Germany

E-mail address: teschl@iram.rwth-aachen.de 